\title{
The contribution of imaging in diagnosis, preoperative assessment, and follow-up of moyamoya disease
}

\author{
A review \\ Susanna Bacigaluppi, M.D., ${ }^{1,3}$ Amir R. Dehdashti, M.D., ${ }^{1}$ Ronit Agid, M.D., ${ }^{2}$ \\ Timo Krings, M.D., Ph.D., ${ }^{2}$ Michael Tymianski, M.D., Ph.D., 1 \\ and David J. Mikulis, M.D., Ph.D. ${ }^{2}$ \\ Divisions of ${ }^{I}$ Neurosurgery and ${ }^{2}$ Neuroradiology, University Health Network, Toronto Western Hospital, \\ University of Toronto, Ontario, Canada; and ${ }^{3}$ Department of Neurosciences and Biomedical Technologies, \\ University of Milan-Bicocca, Milan, Italy
}

The aim of this review was to evaluate the imaging tools used in diagnosis and perioperative assessment of moyamoya disease, with particular attention to the last decade. (DOI: 10.3171.2009.01.FOCUS08296)

KEY WORDS $\bullet$ moyamoya disease $\bullet$ diagnosis $\bullet$
neuroimaging modality $\bullet$ magnetic resonance imaging $\bullet$
computed tomography angiography $\bullet \quad$ catheter angiography
cerebrovascular reactivity

$\mathrm{T}$ HIS review will present different imaging techniques and their contribution to the evaluation of patients with moyamoya disease.

\section{Baseline and Morphological Studies}

\section{The Angiography Method}

The first description of a case of moyamoya disease was based on diagnostic catheter cerebral angiography. ${ }^{43,77,80}$ Catheter angiography still represents the gold standard for moyamoya diagnosis. Three features usually characterize the vascular picture on angiography studies: stenosis of the supraclinoid distal ICA; the development of dilated striate arteries (moyamoya collateral vessels with a characteristic blush); and sparing of the posterior fossa vessels (Fig. 1D, G, and $\mathrm{H}$ ).

\footnotetext{
Abbreviations used in this paper: $\mathrm{ACA}=$ anterior cerebral artery; $\mathrm{BOLD}=$ blood oxygen-level dependent; $\mathrm{CA}=$ carotid artery; $\mathrm{CBF}$ = cerebral blood flow; $\mathrm{CBV}=$ cerebral blood volume; $\mathrm{CCA}=$ common $\mathrm{CA} ; \mathrm{CMRO}_{2}=$ cerebral metabolic rate of oxygen; $\mathrm{CVR}=$ cerebrovascular reactivity; $\mathrm{DS}=$ digital subtraction; $\mathrm{ECA}=$ external CA; EC-IC = extracranial-intracranial; $\mathrm{ETCO}_{2}=$ end-tidal $\mathrm{CO}_{2} ; \mathrm{ICA}=$ internal $\mathrm{CA} ; \mathrm{MCA}=$ middle cerebral artery; MMA = middle meningeal artery; MTT = mean transit time; OEF $=\mathrm{O}_{2}$ extraction fraction; $\mathrm{PCA}=$ posterior cerebral artery; $\mathrm{SAH}=$ subarachnoid hemorrhage; STA = superficial temporal artery; TIA $=$ transient ischemic attack.
}

Stenoses involve the anterior circulation and start in the terminal, supraclinoid part $\left(\mathrm{C}_{1}-\mathrm{C}_{2}\right.$ portion) of the ICA, and subsequently spread to the proximal part of the $\mathrm{ACA}$ and the MCA. It is only in severe cases and latestage disease that the stenotic process involves the PCA. The formation of collateral pathways ${ }^{44}$ initially comprises dilation of intracranial collateral vessels (perforating lenticulostriate arteries, the thalamoperforating arteries, the anterior choroidal, and the posterior pericallosal or splenial arteries). The final stages are characterized principally by the development of collateral vessels from the external circulation (ethmoidal arteries and dural-pial supply). Some authors consider the dilation of the anterior choroidal and posterior communicating arteries predictive for hemorrhage. ${ }^{57}$

Suzuki and Takaku ${ }^{77}$ have described 6 stages of moyamoya progression: 1) narrowing of the CA termination; 2) dilation of the proximal portions of the ACA and MCA with initial basal moyamoya blush; 3) proximal portions of the ACA and MCA are no longer visualized; distal branches are still present due to collateral vessels from the PCA and intensification of the moyamoya blush; 4) minimization of the basal moyamoya network together with progressive occlusion of the ICA, which reaches the origin of the PCA; 5) further reduction of moyamoya vessels, with complete disappearance of the main arteries arising from the CA, continuous decrease of moyamoya collateral vessels that are more limited to the siphon area, 

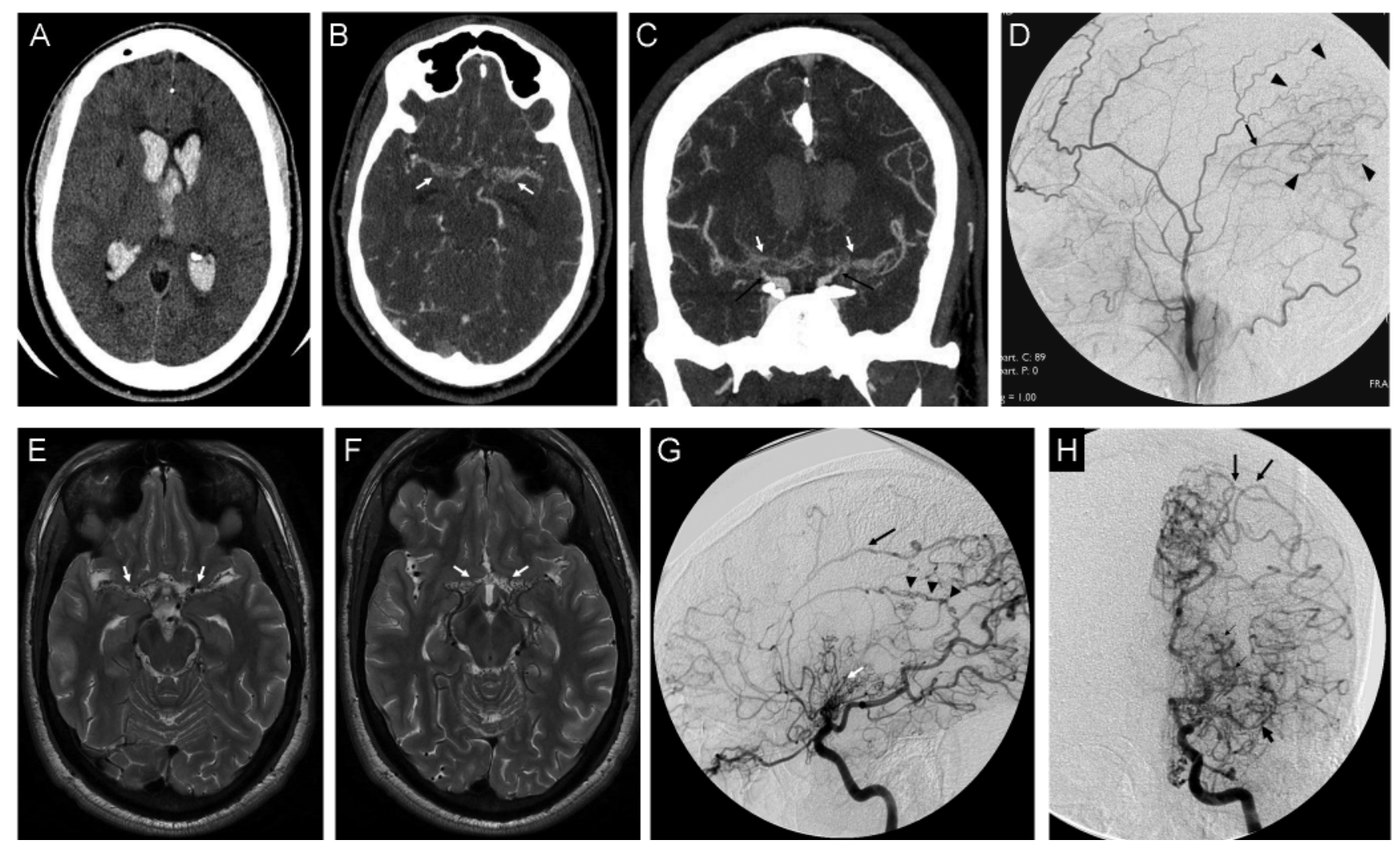

FIG. 1. Neuroimages obtained in a 25-year-old patient presenting with intraventricular hemorrhage. A: Nonenhanced CT scan revealing hemorrhage. B and C: The CT angiography studies of the brain show tight stenosis of the supraclinoid ICAs (black arrows in C) and multiple tiny collateral vessels replacing the $\mathrm{M}_{1}$ segments of the MCAs bilaterally (white arrows in B and C). D: Cerebral angiogram in lateral projection of the left ECA shows collateral flow from the MMA (arrow) opacifying vessels in the posterior MCA territory (arrowheads). E and F: Axial T2-weighted MR images of the brain demonstrating very small multiple tiny flow voids (arrows) in the sylvian fissures representing these same moyamoya collateral vessels. $\mathrm{G}$ and $\mathrm{H}$ : Cerebral angiogram of the left ICA in lateral $(\mathrm{G})$ and anteroposterior $(\mathrm{H})$ projections demonstrates the tight stenosis at the CA terminus involving the $M_{1}$ and $A_{1}$ segments. Leptomeningeal collateral vessels from the PCA territory via watershed into MCA territory are noted (Iong arrows) as well as collateral vessels fed via posterior pericallosal arteries (arrowheads). Classic moyamoya "puff of smoke" collateral vessels originating from perforating arteries are evident (short arrows) and correspond to the findings on CT angiography and MR imaging.

and increased collateral supply from the ECA; and 6) disappearance of the moyamoya blush together with the blood supply from the ICA. At this stage, only the ECA supplies the intracranial circulation.

Angiography might also reveal the presence of aneurysms $^{38}$ that may be related to the increased flow load in relation to the normal vessel diameter in these patients. These aneurysms are found either at the periphery, on the collateral dilated vessels, ${ }^{11,21,46}$ or on the Circle of Willis, ${ }^{36}$ most commonly on the posterior circulation. ${ }^{4,6}$ However, when evaluating the cause of SAH in these patients, one has to also consider hemorrhage from fragile transdural anastomotic vessels..$^{52}$

In extremely rare cases, other vascular anomalies (such as arteriovenous malformations) may be detected in these patients..$^{61,85}$

A further interesting, less investigated angiographic finding is the involvement of the ECA system: stenoses were found (and also histologically proven ${ }^{3,92}$ ) on the STA and on the MMA., ${ }^{8,59,92}$ It was suggested that these findings are associated with lower treatment success rates.

Changes in the angiographic venous filling patterns parallel the arterial changes. In the compensated phases of moyamoya disease, a delayed but increased filling of intracerebral venous drainage is observed, together with an increased but not delayed filling of the external venous draining system. In particular, the dilation of deep intracranial draining vessels, such as the striate veins, reflects the different stages of moyamoya disease. The superior striate vessels dilate together with the increase in basal ganglia moyamoya vessels, and decrease in size in the phase of leptomeningeal compensation. These findings differ from those in atherosclerotic patients, in whom there is no delay in venous filling, but rather territorial rarefaction of venous filling. ${ }^{40}$

\section{The CT Modality}

Usually, the first neuroimaging tool used in diagnosing an emergency patient is a CT scan. Besides evidence of SAH or intraventricular hemorrhage (Fig. 1A), common CT findings in patients with moyamoya disease are those of ischemic cortical stroke. In a series of 18 moyamoya patients, ${ }^{80} 14$ presented with mono- or bilateral cortical or subcortical low-density lesions, consistent with en- 

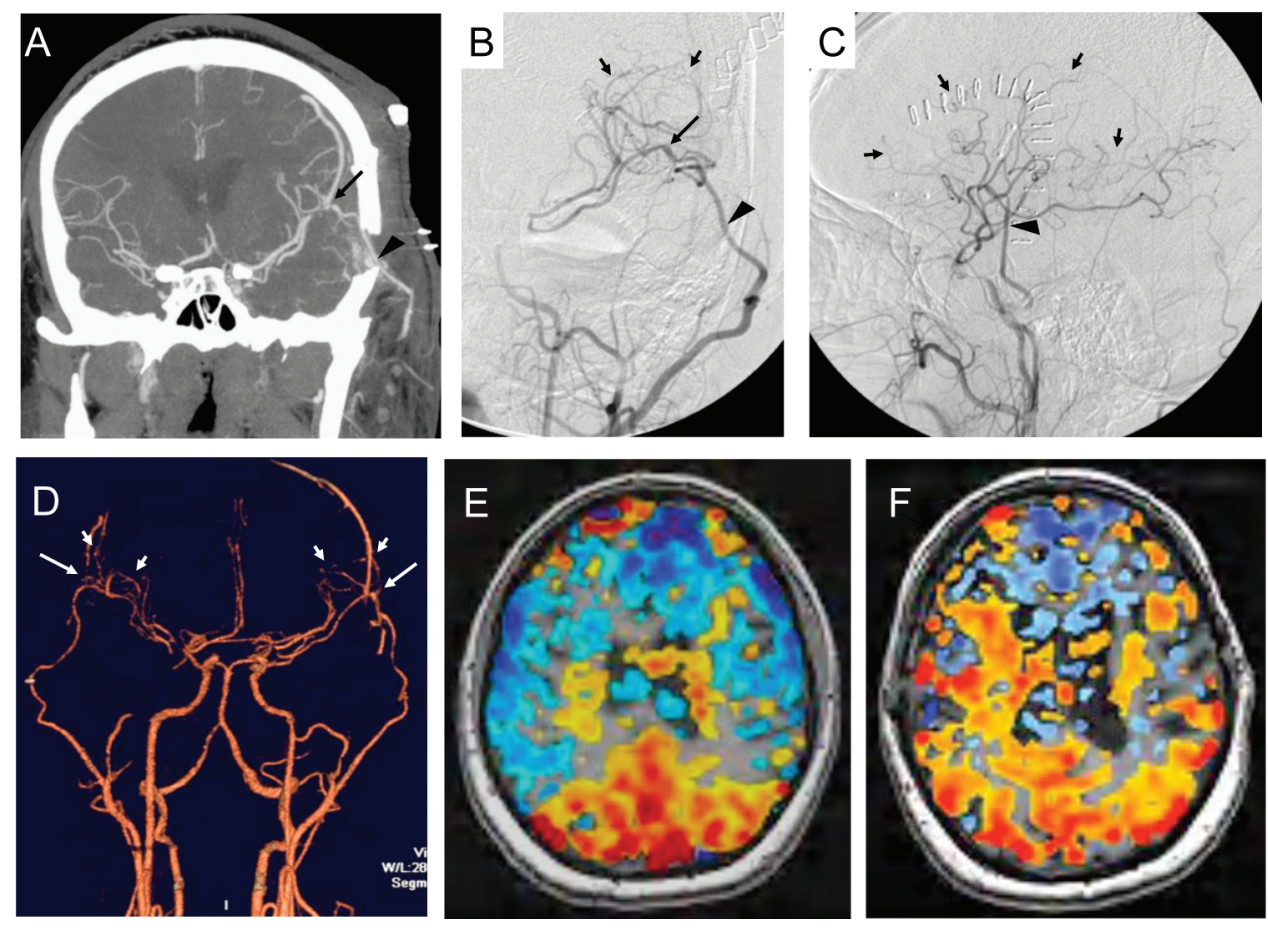

FIG. 2. Neuroimages obtained in a 42-year-old patient presenting with multiple TIAs treated by bilateral EC-IC bypass. A: Coronal maximum intensity projection reconstruction of CT angiography studies, obtained after the first procedure, demonstrates a patent left STA-MCA bypass as it passes through the craniectomy and connects intracranially to a distal branch of the left MCA (arrowhead). B and C: Conventional cerebral angiogram of the left CCA in anteroposterior (B) and lateral (C) projections confirms the patency of this bypass with opacification of vessels of the left MCA territory. D: A 3D reconstruction of a CT angiography study, obtained after performing the second bypass, demonstrates that both STA-MCA anastomoses are patent (arrows). E: The MR imaging CVR studies obtained prior to treatment reveal reduced CVR in bilateral MCA and ACA territories (in blue) but preserved reactivity in the posterior circulation territory (in red and yellow). F: After the bypass procedures there is near normalization of CVR on the right side of the brain and significant improvement on the left. The ACA territory is not significantly changed because the bypass does not enhance perfusion of this region. Designators in this figure include the following: arrowheads denote the STA as it passes through the craniectomy (panels A-C); long arrows denote STA-MCA anastomosis (panels A, B, and D); and short arrows denote MCA branches (panels B-D).

cephalomalacic changes following previous infarctions; ventricular dilation was noted in 9 patients and cortical atrophy in 11 . Only 1 patient in this series with presumable TIA had normal findings on a CT scan of the brain, even after the addition of contrast. The $\mathrm{CT}$ scans obtained after contrast administration revealed enhancing lesions in 3 of 16 patients, and basal ganglia enhancement in 4 of 4 patients undergoing high-load contrast injections. Other features described in the literature include low densities in the basal ganglia in 4 of 6 patients, ${ }^{78}$ and small hyperdense subcortical hyperdensities, referable to calcium deposits, within old infarcted lesions. ${ }^{25}$

\section{The CT Angiography Technique}

The use of CT angiography to image the vessels of the head and neck has evolved rapidly since helical CT introduced the ability to scan quickly and to capture the arterial phase of enhancement. The recent introduction of multidetector row CT scanners such as the 16,64 , or 320 multidetector row scanners enables isotropic imaging, which provides high-resolution $3 \mathrm{D}$ reconstructions and a short acquisition time. This diagnostic tool is especially helpful in patients with suspected vascular abnormalities, particularly in cases of intracerebral hemorrhage. ${ }^{1}$
Stenoses, CA occlusion, moyamoya vessels, and areas of contrast enhancement can be seen. ${ }^{58} \mathrm{~A} \mathrm{CT}$ angiography scan is also a useful device for preoperative planning ${ }^{10}$ and for postsurgical verification of patency of an EC-IC bypass (Fig. 1A and B, Fig. 2A and D).

\section{The MR Imaging Modality}

Types of MR imaging studies include standard acquisitions and MR angiography as well as functional and metabolic studies. Use of MR imaging offers high spatial and temporal resolution without radiation.

In conventional MR imaging, findings in patients with moyamoya disease have been described as primary and secondary. Primary findings include the loss of flow voids of the large arteries, attributable to occlusions of the circle of Willis, and numerous flow voids in the basal cistern and basal ganglia, referable to the augmented vascular network at those sites (Fig. 2E and F). These findings are best seen on T2-weighted images, in which the black vascular flow voids are beautifully contrasted against the bright CSF, a critical observation that, when present and not overlooked, is virtually diagnostic of moyamoya disease. Secondary findings concern signs of cerebral infarction, gliosis, atrophy, and hemorrhage. ${ }^{28}$ 


\section{S. Bacigaluppi et al.}

The FLAIR images offer improved sensitivity to ischemic injury compared with T1- and T2-weighted images and are particularly helpful in the discrimination between subacute and chronic lesions, because the latter usually present with a lower or isointense signal. ${ }^{5}$ In contrast, diffusion-weighted imaging is the most sensitive sequence for identifying acute ischemic stroke, becoming abnormal before FLAIR and T2 images. This technique measures the microscopic motion of water protons and is quantified by measuring the apparent diffusion coefficient, which lumps the effects of random and restricted water motion in tissue. A reduced apparent diffusion coefficient is a sensitive marker of the failure of cellular energy metabolism in the ischemic brain. ${ }^{5}$ Furthermore, signal changes on diffusion-weighted imaging develop in areas of severely reduced perfusion, representing restricted water movement associated with cytotoxic edema.

Asymptomatic microbleeds occur in 15 to $56 \%$ of patients with moyamoya, according to reports in the literature. ${ }^{35,39}$ These lesions are classically present in the periventricular deep white matter, rather than at the corticosubcortical regions or basal ganglia/thalami as described in other diseases. ${ }^{39}$ The authors found nonsignificant differences in the incidence of microhemorrhages between patients with and without antiplatelet therapies. Usually antiaggregants are given to patients with moyamoya who have the occlusive ischemic, nonhemorrhagic disease type. Whether microhemorrhages represent a marker of intracranial hemorrhage has to be clarified. ${ }^{35,56}$

A characteristic sign for moyamoya on MR imaging is leptomeningeal enhancement. It is related to the leptomeningeal flow engorgement, also known as "ivy sign." ${ }^{\prime 1,68}$ This sign is typically detected with T1-weighted contrast-enhanced ${ }^{19,41}$ and FLAIR images, ${ }^{49}$ and can be associated with poor visualization of the MCA. ${ }^{16}$ However, in patients with moyamoya who had large infarcts, poorer visualization of the MCA tree was not associated with leptomeningeal high signal intensity. The following differential diagnoses have to be kept in mind for the "ivy sign": SAH, meningitis, brain tumor or meningeal carcinomatosis, subdural hematoma, CSF hypotension, and hyperbaric $\mathrm{O}_{2}$ therapy..$^{50,81}$

According to the guidelines for the diagnosis and treatment of spontaneous occlusion of the circle of Willis, ${ }^{17}$ angiography is not mandatory for the diagnosis of moyamoya disease, because MR imaging and MR angiography can show all the findings required for diagnosis. Nevertheless, these authors recommend diagnosis made with the aid of MR techniques alone only in childhood cases. The MR imaging should be performed on 1.5-T (or higher) systems, as suggested by the Research Committee for Diagnosis of moyamoya. ${ }^{17}$ Use of 3-T imaging provides higher resolution and can detect even smaller pathological vessels associated with moyamoya. ${ }^{18}$

Different acquisition techniques are available for MR angiography, including 3D time-of-flight MR angiography, phase-contrast MR angiography, and contrast-enhanced MR angiography. Pulse sequence variations have been developed to improve these basic MR angiography methodologies, including turbo MR angiography, which represents 3D fast imaging with steady-state precession and magnetization transfer contrast, tilted optimized nonsaturated excitation, multislab techniques, and zerofilling interpolation, and has been described in a single report as a 98\% accurate technique for diagnosis and assessment of moyamoya disease when compared with conventional angiography. ${ }^{90}$ These authors further analyzed the sensitivity, specificity, and accuracy of these neuroimaging techniques regarding moyamoya features, including stenoocclusive lesions $(100 \%$ for all 3 parameters); basal cerebral moyamoya vessels $(98,100$, and $98 \%$, respectively); and leptomeningeal transdural collateral vessels $(98,100$, and 98\%, respectively) in a sample of 42 patients. Altogether this MR angiographic technique presents acceptable sensitivity (98\%), specificity (100\%), and accuracy (98\%).

Postoperatively, MR angiography has been used for the assessment of bypass filling (with cine-phase MR angiography ${ }^{31}$ ) and for the assessment of changes in the ECAs in cases of indirect revascularization (with time-offlight). ${ }^{30}$ This method was used to evaluate the superficial as well as the deep temporal artery and the MMAs (with time-of-flight). These authors performed a very early postoperative follow-up evaluation and were able to describe dilation of the superficial and of the deep temporal arteries in the first days after surgery.

From a temporal resolution point of view, MR DS angiography with high temporal resolution $(<1$ second $)$ provides images of cerebral hemodynamics that are almost equivalent to intraarterial DS angiography.,26,42 However, spatial resolution is not as high. In the case of tiny leptomeningeal anastomotic vessels in patients with moyamoya disease, these authors were able to recognize only an increased density of contrast rather than individual vessels themselves.

\section{Metabolic Studies}

Several metabolic studies can be used to assess the $\mathrm{CBF}$ reserve in patients with ischemia and to evaluate the need for revascularization.

Basically, 2 types of hemodynamic impairments can be assessed: 1) the response to a vasodilatory stimulus representing a reduced perfusion pressure, either through increased blood volume or through impaired blood flow response; and 2) noninvasive measurements of OEF that detect an increase in $\mathrm{O}_{2}$ extraction. ${ }^{12}$

For the characterization of the level of stenosis in the main arteries, blood volume flow (in $\mathrm{ml} /$ minute), as used by one group, ${ }^{31,66}$ expresses the flow rate in the brain-supplying arteries and seems to be an indicator of vascular territory perfusion.

For the characterization of the parenchymal microcirculation, perfusion studies are performed. The CBF is measured, usually as the quantity of blood (in $\mathrm{ml}$ ) perfusing a volume of brain (100 g) per unit of time (in minutes).

There are other parameters linked to CBF: the CBV is the amount of blood in a given volume of brain, whereas the MTT represents the time the blood takes to pass through the brain tissue. The 2 parameters are linked by the following relationship: $\mathrm{CBF} \times \mathrm{MTT}=\mathrm{CBV} .{ }^{33}$ The $\mathrm{CBF}$ and $\mathrm{CBV}$ values are tightly coupled. ${ }^{23}$ 


\section{Review of imaging modalities used to assess moyamoya disease}

Numerous factors affect $\mathrm{CBF}$, including arterial perfusion pressure, intracranial pressure, blood viscosity, $\mathrm{PaCO}_{2}, \mathrm{pH}$, and $\mathrm{O}_{2}$.

According to our current understanding of the brain microvasculature, there is autoregulation of $\mathrm{CBF}$ at the parenchymal level influenced by locoregional $\mathrm{pH}$, nitric oxide, and other paracrine mediators through relaxation of the arteriolar smooth-muscle tone, permitting fine-tuning of blood flow. Assessment of autoregulation capacity presumes that blood flow within the microcirculation distal to stenotic large feeding vessels is maintained through the relaxation of arteriolar smooth muscle. In the case of patients with uncompensated moyamoya disease, once the maximum arteriolar dilation is reached, any further narrowing of the feeding vessels leads to decreased blood flow and ischemia.

Most imaging techniques used for the acquisition of CVR data require the administration of agents that can change resting blood flow.

Acetazolamide challenge is commonly used to assess cerebrovascular autoregulation, and is particularly useful in the assessment of chronic ischemic disease. ${ }^{22,29}$ This drug is an inhibitor of carbonic anhydrase and evokes a shift in the cerebral acid-base balance toward carbonic acidosis, thus increasing $\mathrm{CBF}^{7,20}$

Another technique that can be used for the same purpose is $\mathrm{ETCO}_{2}$ manipulation. This technique has been assessed in combination with $\mathrm{Xe}-\mathrm{CT}$ and, more recently, with BOLD MR in the study of patients with moyamoya disease $^{54}$ (Fig. 2E and F).

With most techniques (for example, BOLD MR and $\mathrm{Xe}-\mathrm{CT}$ ), cerebrovascular reserve capacity maps are calculated as the absolute change of $\mathrm{CBF}$ from the baseline to the stimulation scan.

\section{The Xe-CT Technique}

The Xe-CT technique is used to assess hemodynamic stress by measuring CVR with a tolerance test (acetazolamide or $\mathrm{CO}_{2}$ ). Stable xenon is a radiopaque diffusible gas that progressively enters tissues based on blood flow. This examination relies on dynamic CT scanning during inhalation of a gas mixture containing stable xenon and $\mathrm{O}_{2}$. The Xe-CT technique requires patient cooperation and expensive add-on equipment. There can be associated side effects, including respiratory failure, headaches, nausea, vomiting, and convulsions. A xenon-inhalation assessment can be performed either in combination with acetazolamide $^{48,53}$ or with ETCO ETnipulation. $^{32}$ Cerebral blood flow maps can thus be obtained, processed with correction factors for $\mathrm{ETCO}_{2}, \mathrm{CO}_{2}$ variance, and hematocrit. A CBF value of $<30 \mathrm{ml} / \mathrm{min} / 100 \mathrm{~cm}^{3}$ for adults or $<40 \mathrm{ml} / \mathrm{min} / 100 \mathrm{~cm}^{3}$ for children is defined as an ischemic region, because normal $\mathrm{CBF}$ values in children are $20-60 \%$ greater than in adults. ${ }^{9}$ An increase of blood flow after acetazolamide of $<10 \mathrm{ml} / \mathrm{min} / 100 \mathrm{~cm}^{3}$ is considered to be the threshold. ${ }^{64}$ However, for treatment decisionmaking purposes, these authors used the threshold value of 0 , because areas with a value $<0$ were found to represent areas with high stroke rates in a prospective study ${ }^{93}$ and to improve after indirect EC-IC bypass surgery. ${ }^{64,65}$

\section{The Perfusion CT Technique}

As opposed to Xe-CT, perfusion $\mathrm{CT}$ relies less on patient collaboration and is very fast..$^{90}$ This modality is based on the sequential acquisition of axial brain CT sections during intravenous contrast injection. Perfusion CT data consist of contrast enhancement profiles with spatial and temporal coordinates. ${ }^{89}$

Nevertheless, CBF measurements with CT perfusion require an appropriate choice of a reference artery for flow calculation. The arterial input to the pathological tissue must be known to quantitate CBF accurately. Because these arteries can be severely narrowed or absent in moyamoya, determining the arterial input is difficult. This point is critical in patients with moyamoya disease, ${ }^{73}$ because arteries like the ACA or the MCA may not be available for input function assessment. In these patients, vessels of the posterior circulation are a possible alternative. This issue explains the limited number of moyamoya papers in the literature in which CT perfusion has been used. A recent study ${ }^{37}$ (16 patients with moyamoya disease and 17 healthy control volunteers) shows that while the normalized baseline CT perfusion data weakly correlate with angiography, acetazolamide-challenged CT perfusion significantly correlates with the angiographic stage. Delay-insensitive perfusion techniques are now being developed to address problems related to different arrival times of contrast agents in impaired vascular territories.

\section{The PET Modality}

Positron emission tomography studies for hemodynamic assessment are usually performed with the following tracers: $\mathrm{C}^{15} \mathrm{O}$ PET for $\mathrm{CBV}$ assessment, $\mathrm{H}_{2}{ }^{15} \mathrm{O}$ for $\mathrm{CBF}$, and ${ }^{15} \mathrm{O}_{2}$ to measure $\mathrm{OEF}$ and $\mathrm{CMRO}_{2} \cdot{ }^{34}$

Nariai et al. ${ }^{62}$ identified differences in hemodynamic characteristics among the various clinical presentations of moyamoya disease. In this study, patients were classified as nonsymptomatic, those with hemorrhage, and those with ischemic symptoms. The latter group was subdivided into patients who presented with TIA only, patients with TIA and infarct TIA, and patients who suffered a permanent deficit following an infarction. Using PET and comparing results to healthy controls, the authors found that $\mathrm{CBF}$ in the patient groups classified as nonsymptomatic, TIA, and hemorrhage was not significantly lower than in healthy controls in any region. The CBV in the TIA and infarct TIA groups was significantly higher than in controls. The OEF in the frontal, parietal, and temporal cortex was significantly higher in the infarct TIA group than in the controls. Patients in the hemorrhage and permanent deficit groups had decreased metabolism with normal OEF. A paradoxical steal phenomenon is observed when the vascular bed is maximally dilated and autoregulation is completely lost. ${ }^{63,93}$ It is associated with increased CBV and increased OEF. Interestingly, increased CBV and a normal or mildly increased OEF is rarely observed in atherosclerotic patients, ${ }^{13}$ possibly due to the ability to invoke circle of Willis collateral vessels, which are lost in patients with moyamoya secondary to involvement of these collateral vessels by the disease process. Patients 


\section{S. Bacigaluppi et al.}

with moyamoya are therefore more dependent on a less effective pial collateral circulation for maintenance of flow. Patients in the infarct TIA group, who showed a marked increase in the $\mathrm{OEF}$, were considered to be at risk for further deterioration. In these patients the MTT (which usually has an inverse relationship with cerebral perfusion pressure), was larger. Evidence of the effectiveness of revascularization was nicely shown in a series of 23 patients who were treated either with synangiosis or with bypass. Postoperative follow-up PET studies showed improved $\mathrm{CO}_{2}$ reactivity in this group. ${ }^{45}$

The PET modality is one of the most reliable assessment tools because it provides quantitative $\mathrm{CBF}, \mathrm{CBV}$, $\mathrm{OEF}$, and $\mathrm{CMRO}_{2}$ information; however, there are still several limits. First, a cyclotron is required near the PET scan to deliver rapidly decaying tracers. Second, PET is associated with delivery of ionizing radiation: a complete 2D PET hemodynamic assessment (with CBF, CBV, $\mathrm{CMRO}_{2}$, and $\mathrm{OEF}$ ) is associated with an effective dose of $8.9 \mathrm{mSv}$ to the patient. Third, the number of available PET scanners is far less than MR, CT, or SPECT systems, although 3D PET is becoming available, and it offers the advantage of comparable accuracy with shorter scan duration and lower tracer doses. The radiation dose is reduced to approximately one-third: for example, for ${ }^{15} \mathrm{O}_{2}$ the effective dose becomes 1.2 instead of $4.7 \mathrm{mSv}$, and a complete hemodynamic study is associated with an effective dose estimate of approximately $2.2 \mathrm{mSv} .^{34}$

\section{The SPECT Method}

This methodology has also been used to assess regional $\mathrm{CBF}$ and CVR in moyamoya studies. It is performed using a radioactive blood tracer ( ${ }^{123} \mathrm{I}-\mathrm{IMP}-\mathrm{ARG} \mathrm{G}^{60}$ or ${ }^{99 \mathrm{~m}} \mathrm{Tc}-\mathrm{HMPAO}^{27,82}$ ) that is able to cross the blood-brain barrier and to remain fixed for enough time to allow the extracranial tomographic imaging of the gamma emission. Two separate imaging sessions (baseline and acetazolamide challenge) are required, usually separated by 24 hours to enable tracer washout between sessions. The tracer is deposited in proportion to blood flow, predominantly during the first pass through the microcirculation, and it is therefore a marker of flow without providing actual blood flow in terms of $\mathrm{ml} / 100 \mathrm{~g} / \mathrm{min}$. Also, the use of ${ }^{123}$ I-iomazenil has been described in patients with moyamoya disease, to evaluate neuronal damage. ${ }^{74}$

\section{The MR Imaging-Based Techniques}

For evaluation of stenosis in larger vessels in patients with moyamoya disease, blood flow volume studies in which dynamic 2D cine-phase contrast MR imaging is used have demonstrated a significant decrease in ICA blood flow volume associated with an impressive increase (nearly 2.5 times) in basilar artery blood flow. ${ }^{66}$ Flow in the ICA was measured at $118 \pm 64 \mathrm{ml} / \mathrm{minute}$ in patients with moyamoya versus $254 \pm 25 \mathrm{ml} /$ minute in age-matched controls. Basilar artery flow measured 434 $\pm 166 \mathrm{ml} / \mathrm{minute}$ in patients with moyamoya versus $173 \pm$ $13 \mathrm{ml} / \mathrm{minute}$ in controls.

Two methods are available for assessment of brain perfusion by MR imaging: 1) the "dynamic" susceptibil- ity contrast technique uses principles such as indicator dilution methods in which a decrease in T2 or T2* MR signal intensity is related to the passage of a paramagnetic contrast agent through the capillary bed; and 2) the "arterial spin labeling" technique uses inversion or saturation pulses that label intravascular blood water protons before delivery into the brain slice of interest. ${ }^{33,75}$

An emerging technique for the assessment of CVR is BOLD MR imaging during manipulation of end-tidal $\mathrm{PCO}_{2}{ }^{51,67,71}$ This method does not require the use of a pharmacological agent to change resting blood flow; instead, stimulus application is obtained through a rebreathing circuit in which end-tidal $\mathrm{PCO}_{2}$ levels are manipulated between normocapnea and hypercapnea for measuring vasodilatory reserve, and between normocapnea and hypocapnea to measure vasoconstrictive reserve. This is facilitated using a rebreathing device that permits precision control of $\mathrm{ETCO}_{2}$ in square wave patterns. ${ }^{84}$ At the same time, BOLD MR sequences identical to those used for functional MR studies are obtained. The CVR maps generated through this method provide spatial, quantitative maps of impaired CVR in terms of percent BOLD signal change per $\mathrm{mm} \mathrm{Hg}$ change in $\mathrm{ETCO}_{2}$. Thus, the MR imaging method is threshold independent for determining the presence and spatial extent of exhausted autoregulation. Negative CVR responses (decreases in the BOLD MR imaging signal) are observed in patients with uncompensated moyamoya disease, indicating the presence of the steal phenomenon. Under these conditions, hypercapnia diverts blood away from maximally dilated vascular beds to unimpaired beds that retain the capacity for vasodilation..$^{54,69,84}$ The advantages of this technique are the following: 1) measurements occur at the tissue level, providing spatial maps of impaired CVR; 2) the method does not require measurement of a vascular input function, which can be unreliable in patients with bilateral stenoses; 3) this technique can be added to conventional MR studies already used in the routine workup for these patients; and 4) the technique is safe, with no exposure to ionizing radiation, no injection is needed, and the range of $\mathrm{ETCO}_{2}$ required is within that achieved by patients in daily life situations $(30-50 \mathrm{~mm} \mathrm{Hg}){ }^{57}$

Proton-beam MR spectroscopy also deserves a brief note. This MR imaging technique provides insight into different brain metabolites. Interestingly, some authors have noted that patients with moyamoya disease have reduced levels of choline, creatinine, and $N$-acetylaspartate in the affected brain. A relative increase in these metabolites is then noted 6 months after revascularization surgery. ${ }^{76}$ Many authors consider $N$-acetylaspartate to be an important marker for healthy neurons. ${ }^{57}$

\section{The Ultrasonography Modality}

The ultrasonography modality combines both static and dynamic information, and provides supplementary data regarding quantitative flow characteristics (flow velocity), including the ratio between systolic and enddiastolic velocity, flow resistance, and vessel pulsatility. There are several studies confirming the potential of transcranial power Doppler and color Doppler ultrasonography in the detection of arteries that appear occluded on 
Review of imaging modalities used to assess moyamoya disease

TABLE 1: Effective radiation dose, acquisition time, and spatial resolution of different brain imaging modalities*

\begin{tabular}{|c|c|c|c|c|c|c|c|}
\hline \multirow[b]{2}{*}{ Factor } & \multicolumn{7}{|c|}{ Imaging Modality } \\
\hline & CT & 2D DSA & CTA & $\mathrm{Xe}-\mathrm{CT}$ & $\begin{array}{l}\text { Perfusion } \\
\text { CT }\end{array}$ & PET & SPECT \\
\hline $\begin{array}{l}\text { effective dose } \\
\text { exposure }\end{array}$ & $\begin{array}{l}1.1-2.5 \\
m S v^{83,87}\end{array}$ & $10.5 \mathrm{mSv}^{15}$ & 3.57-5.73 mSv ${ }^{91}$ & $3.5-10 \mathrm{mSv}^{88}$ & $2-3 \mathrm{mSv}^{88}$ & $2.2-8.9 \mathrm{mSv}^{34}$ & $\begin{array}{l}3.5-12 \\
\mathrm{mSv} 88\end{array}$ \\
\hline data acquisition time & $0.5 \mathrm{~min}$ & $15 \mathrm{~min}$ & $20-70 \sec ^{24}$ & $4 \min ^{88}$ & $1-2 \min ^{88}$ & $10 \min ^{34}$ & $10 \min ^{88}$ \\
\hline spatial resolution & $1 \mathrm{~mm}$ & $0.2 \mathrm{~mm}^{86}$ & $0.6-1 \mathrm{~mm}^{24}$ & $4 \mathrm{~mm}^{88}$ & $1-2 m^{88}$ & $4-6 \mathrm{~mm}^{88}$ & $4-6 \mathrm{~mm}^{88}$ \\
\hline
\end{tabular}

* Data were obtained either from recent literature or from our neuroradiological service. Variation in the radiation doses will occur, based on different manufactures and user-selectable parameters (such as $\mathrm{kV}$ and $\mathrm{mA}$ for the $\mathrm{x}$-ray techniques). Abbreviations: CTA = CT angiography; DSA = DS angiography.

DS angiography ${ }^{72}$ or on MR angiography. ${ }^{2,14,47}$ According to some authors, ${ }^{73}$ ultrasonography was also able to reveal alterations of vessels that appear normal on angiography, while at the same time not all angiographic features could be found on ultrasound studies. This seems to prove the complementarities of different imaging tools and the need for interpretation of nonoverlapping findings.

Takase et al. ${ }^{79}$ stress the importance of performing transcranial Doppler tracing over the entire length of the basilar arteries in these patients. They classified the CBF velocity patterns into 3 types: 1) the high-high flow pattern, commonly seen in younger patients, in whom high flow velocities exist with high flow, but with moderategrade stenosis in the ICA or MCA, in the absence of proper collateralization; 2) the high-low flow pattern, in which higher-grade stenoses are associated with high velocity but lower overall flow; and 3) the low-low flow pattern, in which velocity and flow are low secondary to the highest degree of stenosis.

Doppler studies provide us with further information about features distinguishing the CCA and ICA from the STA: pulsatility, flow resistance, and systolic/end diastolic velocity were elevated and flow velocity was decreased in the former, whereas in the latter (the STA), the pulsatility, flow resistance, and systolic/end diastolic values were lower and flow velocity was greater in patients with moyamoya disease than in controls. ${ }^{72}$

Compared with the color Doppler method, power Doppler is relatively independent from the insonation angle, is not subject to aliasing, and is associated with relatively low background noise, thus offering an increase in sensitivity of approximately $10-15 \mathrm{~dB}$. This accounts for the good sensitivity in the detection of low-flow areas and of parts of the intracranial collateral network, ${ }^{55,72}$ with a spatial resolution $>1 \mathrm{~mm}$. It has been described as a valid method for diagnosis ${ }^{73}$ and for the evaluation of direct (bypass patency) and indirect (neoangiogenesis) revascularization, with a good interobserver agreement for a 3 -grade scale. ${ }^{70}$

\section{Conclusions}

We have summarized the most recent imaging applications for the study of patients with moyamoya disease. Full characterization of the disease process requires a combined approach that assesses the anatomical and morphological features of the vascular network, the degree to which flow compensation is invoked through development of collateral vessels and lowering of vascular resistance via relaxation of tone in the peripheral resistance vessels (autoregulation), and the extent of metabolic impairment through measurement of $\mathrm{O}_{2}$ consumption. No single imaging tool is capable of providing all of this information.

For assessing the vascular anatomy, conventional DS angiography continues to offer the highest spatial and temporal resolution; however, MR and CT angiography are adequate and are now commonly used for diagnosis and management.

Several methods are available for assessing autoregulation, each with certain limitations. Ultrasonography cannot provide maps of the extent and degree of CVR impairment at the tissue level. Acetazolamide SPECT and $\mathrm{Xe}-\mathrm{CT}$ scans have been considered gold standards for the clinical assessment of CVR deficits. However, they suffer from reproducibility differences due to variations in patient response to acetazolamide. The PET method can be used to measure both CVR and tissue respiration, but requires a cyclotron on site. The BOLD MR modality with controlled $\mathrm{ETCO}_{2}$ is very promising for CVR assessment, with the following advantages: 1) it is easily implemented on all modern MR imaging systems; 2) it is threshold independent for quantitative mapping of impaired autoregulation with vascular steal; and 3) it is not associated with ionizing radiation. Table 1 summarizes information worth considering when using methods based on ionizing radiation.

Currently at our institution we use the following neuroimaging tools: because the detection of diminutive circle of Willis vessels associated with innumerable small flow voids in the basal cisterns and basal ganglia on T2weighted MR imaging sequences often establishes the diagnosis of moyamoya, we mostly use CT angiography to detail vascular morphology, and we use BOLD MR imaging with control of the $\mathrm{ETCO}_{2}$ for perioperative and follow-up assessment of CVR.

Finally, in our clinical practice we always treat symptomatic hemispheres showing impaired CVR. Interestingly, we have frequently observed that treatment of one side is often also associated with contralateral CVR im- 


\section{S. Bacigaluppi et al.}

provement. Our first-choice treatment modality is always the STA-MCA bypass. Only in the absence of suitable recipient vessels do we perform myosynangiosis. For MCA revascularization, we usually select the branch with the largest diameter. When stenosis includes the $\mathrm{M}_{1}$ bifurcation, we analyze the spatial information in the CVR map to target the branch supplying the area with the greatest steal. Future outcome studies will allow us to determine the impact of using these CVR maps for surgical guidance.

\section{Disclaimer}

The authors report no conflict of interest concerning the materials or methods used in this study or the findings specified in this paper.

\section{References}

1. Agid R, Lee SK, Willinsky RA, Farb RI, ter Brugge KG: Acute subarachnoid hemorrhage: using 64 slice multidetector CT angiography to "triage" patients' treatment. Neuroradiology 48:787-794, 2006

2. Aoki S, Yoshikawa T, Hori M, Ishigame K, Nambu A, Kumagai $\mathrm{H}$, et al: Two-dimensional thick-slice MR digital subtraction angiography for assessment of cerebrovascular occlusive diseases. Eur Radiol 10:1858-1864, 2000

3. Aoyagi M, Fukai N, Yamamoto M, Nakagawa K, Matsushima Y, Yamamoto K: Early development of intimal thickening in superficial temporal arteries in patients with moyamoya disease. Stroke 27:1750-1754, 1996

4. Arita K, Kurisu K, Ohba S, Shibukawa M, Kiura H, Sakamoto $\mathrm{S}$, et al: Endovascular treatment of basilar tip aneurysms associated with moyamoya disease. Neuroradiology 45:441-444, 2003

5. Barnett HJM, Mohr JP, Stein BM, Yatsu F: Stroke: Pathophysiology, Diagnosis and Management, ed 3. Philadelphia: Churchill Livingstone, 1998, pp 229-234

6. Bhattacharjee AK, Tamaki N, Minami H, Ehara K: Moyamoya disease associated with basilar tip aneurysm. J Clin Neurosci 6:268-271, 1999

7. Bickler PE, Litt L, Banville DL, Severinghaus JW: Effects of acetazolamide on cerebral acid-base balance. J Appl Physiol 65:422-427, 1988

8. Chen ST, Liu YH, Hsu CY, Hogan EL, Ryu SJ: Moyamoya disease in Taiwan. Stroke 19:53-59, 1988

9. Chiron C, Raynaud C, Mazière B, Zilbovicius M, Laflamme $\mathrm{L}$, Masure MC, et al: Changes in regional cerebral blood flow during brain maturation in children and adolescents. J Nucl Med 33:696-703, 1992

10. Coppens JR, Cantando JD, Abdulrauf SI: Minimally invasive superficial temporal artery to middle cerebral artery bypass through an enlarged bur hole: the use of computed tomography angiography neuronavigation in surgical planning. J Neurosurg 109:553-558, 2008

11. da Costa LB, Valiante T, ter Brugge KG, Tymianski M: Anterior ethmoidal artery aneurysm and intracerebral hemorrhage. AJNR Am J Neuroradiol 27:1672-1674, 2006

12. Derdeyn CP, Grubb RL Jr, Powers WJ: Cerebral hemodynamic impairment: methods of measurement and association with stroke risk. Neurology 53:251-259, 1999

13. Derdeyn CP, Videen TO, Yundt KD, Fritsch SM, Carpenter DA, Grubb RL, et al: Variability of cerebral blood volume and oxygen extraction: stages of cerebral haemodynamic impairment revisited. Brain 125:595-607, 2002

14. Devuyst G, Cals N, de Borchgrave V, Bara P, Vandooren M: Advantages of transcranial power duplex imaging after contrast injection to detect low flow in a moyamoya syndrome. Stroke 30:897-899, 1999
15. Feygelman VM, Huda W, Peters KR: Effective dose equivalents to patients undergoing cerebral angiography. AJNR Am J Neuroradiol 13:845-849, 1992

16. Fujiwara H, Momoshima S, Kuribayashi S: Leptomeningeal high signal intensity (ivy sign) on fluid-attenuated inversionrecovery (FLAIR) MR images in moyamoya disease. Eur J Radiol 55:224-230, 2005

17. Fukui M: Guidelines for the diagnosis and treatment of spontaneous occlusion of the circle of Willis ('moyamoya' disease). Research Committee on Spontaneous Occlusion of the Circle of Willis (Moyamoya Disease) of the Ministry of Health and Welfare, Japan. Clin Neurol Neurosurg 99 (2 Suppl): S238-S240, 1997

18. Fushimi Y, Miki Y, Kikuta K, Okada T, Kanagaki M, Yamamoto A, et al: Comparison of 3.0- and 1.5-T three-dimensional time-of-flight MR angiography in moyamoya disease: preliminary experience. Radiology 239:232-237, 2006

19. Gaa J, Weidauer S: Unusual differential diagnosis of leptomeningeal enhancement: moyamoya disease. J Neurol Neurosurg Psychiatry 75:1170, 2004

20. Gotoh F, Shinohara Y: Role of carbonic anhydrase in chemical control and autoregulation of cerebral circulation. Int $\mathbf{J}$ Neurol 11:219-227, 1977

21. Grabel JC, Levine M, Hollis P, Ragland R: Moyamoya-like disease associated with a lenticulostriate region aneurysm. Case report. J Neurosurg 70:802-803, 1989

22. Grossmann WM: Koeberle: The dose-response relationship of acetazolamide on the cerebral blood flow in normal subjects. Cerebrovasc Dis 10:65-69, 2000

23. Grubb RL Jr, Hernandez-Perez MJ, Raichle ME, Phelps ME: The effects of iodinated contrast agents on autoregulation of cerebral blood flow. Stroke 5:155-160, 1974

24. Hallett RL, Fleischmann D: Tools of the trade for CTA: MDCT scanners and contrast medium injection protocols. Tech Vasc Interv Radiol 9:134-142, 2006

25. Handa J, Nakano Y, Okuno T, Komuro H, Hojyo H, Handa $\mathrm{H}$ : Computerized tomography in Moyamoya syndrome. Surg Neurol 7:315-319, 1977

26. Hans FJ, Reinges MH, Reipke P, Reinacher P, Krings T: Clinical applications of 2-D dynamic contrast-enhanced MR subtraction angiography in neurosurgery-preliminary results. Zentralbl Neurochir 66:170-179, 2005

27. Harada M, Miyoshi H, Uno M, Okada T, Hisaoka S, Hori A, et al: Neuronal impairment of adult moyamoya disease detected by quantified proton MRS and comparison with cerebral perfusion by SPECT with tc-99m HM-PAO: a trial of clinical quantification of metabolites. J Magn Reson Imaging 10:124-129, 1999

28. Hoffman HJ: Moyamoya disease and syndrome. Clin Neurol Neurosurg 99 (2 Suppl):S39-S44, 1997

29. Højer-Pedersen E: Effect of acetazolamide on cerebral blood flow in subacute and chronic cerebrovascular disease. Stroke 18:887-891, 1987

30. Honda M, Kitagawa N, Tsutsumi K, Morikawa M, Nagata I, Kaminogo M: Magnetic resonance angiography evaluation of external carotid artery tributaries in moyamoya disease. Surg Neurol 64:325-330, 2005

31. Horn P, Vajkoczy P, Schmiedek P, Neff W: Evaluation of extracranial-intracranial arterial bypass function with magnetic resonance angiography. Neuroradiology 46:723-729, 2004

32. Horn P, Vajkoczy P, Thomé C, Muench E, Schilling L, Schmiedek P: Xenon-induced flow activation in patients with cerebral insult who undergo xenon-enhanced CT blood flow studies. AJNR Am J Neuroradiol 22:1543-1549, 2001

33. Huisman TA, Sorensen AG: Perfusion-weighted magnetic resonance imaging of the brain: techniques and application in children. Eur Radiol 14:59-72, 2004

34. Ibaraki M, Miura S, Shimosegawa E, Sugawara S, Mizuta T, Ishikawa A, et al: Quantification of cerebral blood flow and 
Review of imaging modalities used to assess moyamoya disease

oxygen metabolism with 3-dimensional PET and 15O: validation by comparison with 2-dimensional PET. J Nucl Med 49:50-59, 2008

35. Ishikawa T, Kuroda S, Nakayama N, Terae S, Kudou K, Iwasaki Y: Prevalence of asymptomatic microbleeds in patients with moyamoya disease. Neurol Med Chir (Tokyo) 45:495500,2005

36. Iwama T, Todaka T, Hashimoto N: Direct surgery for major artery aneurysm associated with moyamoya disease. Clin Neurol Neurosurg 99 (2 Suppl):S191-S193, 1997

37. Kang KH, Kim HS, Kim SY: Quantitative cerebrovascular reserve measured by acetazolamide-challenged dynamic CT perfusion in ischemic adult Moyamoya disease: initial experience with angiographic correlation. AJNR Am J Neuroradiol 29:1487-1493, 2008

38. Kawaguchi S, Sakaki T, Morimoto T, Kakizaki T, Kamada $\mathrm{K}$ : Characteristics of intracranial aneurysms associated with moyamoya disease. A review of 111 cases. Acta Neurochir (Wien) 138:1287-1294, 1996

39. Kikuta K, Takagi Y, Nozaki K, Hanakawa T, Okada T, Mikuni $\mathrm{N}$, et al: Asymptomatic microbleeds in moyamoya disease: T2*-weighted gradient-echo magnetic resonance imaging study. J Neurosurg 102:470-475, 2005

40. Kimura H, Oka K, Ikeda K, Yamamoto M, Tomonaga M: The clinical significance of cerebral veins in moyamoya disease. Clin Neurol Neurosurg 99 (2 Suppl):S90-S95, 1997

41. Komiyama M, Nakajima H, Nishikawa M, Yasui T, Kitano S, Sakamoto H: Leptomeningeal contrast enhancement in moyamoya: its potential role in postoperative assessment of circulation through the bypass. Neuroradiology 43:17-23, 2001

42. Krings T, Hans F: New developments in MRA: time-resolved MRA. Neuroradiology 46 (2 Suppl):S214-S222, 2004

43. Kuroda S, Houkin K: Moyamoya disease: current concepts and future perspectives. Lancet Neurol 7:1056-1066, 2008

44. Kuroda S, Ishikawa T, Houkin K, Nanba R, Hokari M, Iwasaki Y: Incidence and clinical features of disease progression in adult moyamoya disease. Stroke 36:2148-2153, 2005

45. Kuwabara Y, Ichiya Y, Sasaki M, Yoshida T, Masuda K, Ikezaki K, et al: Cerebral hemodynamics and metabolism in moyamoya disease-a positron emission tomography study. Clin Neurol Neurosurg 99 (2 Suppl):S74-S78, 1997

46. Lee JK, Lee JH, Kim SH, Lee MC: Distal anterior choroidal artery aneurysm in a patient with moyamoya disease: case report. Neurosurgery 48:222-225, 2001

47. Lee YS, Jung KH, Roh JK: Diagnosis of moyamoya disease with transcranial Doppler sonography: correlation study with magnetic resonance angiography. J Neuroimaging 14:319323,2004

48. Leinsinger G, Piepgras A, Einhäupl K, Schmiedek P, Kirsch CM: Normal values of cerebrovascular reserve capacity after stimulation with acetazolamide measured by xenon 133 single-photon emission CT. AJNR Am J Neuroradiol 15:13271332,1994

49. Maeda M, Tsuchida C: "Ivy sign" on fluid-attenuated inversion-recovery images in childhood moyamoya disease. AJNR Am J Neuroradiol 20:1836-1838, 1999

50. Maeda M, Yagishita A, Yamamoto T, Sakuma H, Takeda K: Abnormal hyperintensity within the subarachnoid space evaluated by fluid-attenuated inversion-recovery MR imaging: a spectrum of central nervous system diseases. Eur Radiol 13 (4 Suppl):L192-L201, 2003

51. Mandell DM, Han JS, Poublanc J, Crawley AP, Stainsby JA, Fisher JA, et al: Mapping cerebrovascular reactivity using blood oxygen level-dependent MRI in patients with arterial steno-occlusive disease: comparison with arterial spin labeling MRI. Stroke 39:2021-2028, 2008

52. Marushima A, Yanaka K, Matsuki T, Kojima H, Nose T: Subarachnoid hemorrhage not due to ruptured aneurysm in moyamoya disease. J Clin Neurosci 13:146-149, 2006
53. McAuley DJ, Poskitt K, Steinbok P: Predicting stroke risk in pediatric moyamoya disease with xenon-enhanced computed tomography. Neurosurgery 55:327-332, 2004

54. Mikulis DJ, Krolczyk G, Desal H, Logan W, Deveber G, Dirks $\mathrm{P}$, et al: Preoperative and postoperative mapping of cerebrovascular reactivity in moyamoya disease by using blood oxygen level-dependent magnetic resonance imaging. J Neurosurg 103:347-355, 2005

55. Morgenstern C, Griewing B, Müller-Esch G, Zeller JA, Kessler C: Transcranial power-mode duplex ultrasound in two patients with moyamoya syndrome. J Neuroimaging 7:190192, 1997

56. Mori N, Miki Y, Kikuta K, Fushimi Y, Okada T, Urayama S, et al: Microbleeds in moyamoya disease: susceptibility-weighted imaging versus $\mathrm{T} 2 *$-weighted imaging at 3 Tesla. Invest Radiol 43:574-579, 2008

57. Morioka M, Hamada J, Kawano T, Todaka T, Yano S, Kai Y, et al: Angiographic dilatation and branch extension of the anterior choroidal and posterior communicating arteries are predictors of hemorrhage in adult moyamoya patients. Stroke 34:90-95, 2003

58. Murai Y, Takagi R, Ikeda Y, Yamamoto Y, Teramoto A: Three-dimensional computerized tomography angiography in patients with hyperacute intracerebral hemorrhage. J Neurosurg 91:424-431, 1999

59. Murphy MJ: Progressive vascular changes in Moyamoya syndrome. Stroke 11:656-658, 1980

60. Nakagawara J, Takeda R, Suematsu K, Nakamura J: Quantification of regional cerebral blood flow and vascular reserve in childhood moyamoya disease using [123I]IMP-ARG method. Clin Neurol Neurosurg 99 (2 Suppl):S96-S99, 1997

61. Nakashima T, Nakayama N, Furuichi M, Kokuzawa J, Murakawa T, Sakai N: Arteriovenous malformation in association with moyamoya disease. Report of two cases. Neurosurg Focus 5(5):E6, 1998

62. Nariai T, Matsushima Y, Imae S, Tanaka Y, Ishii K, Senda $\mathrm{M}$, et al: Severe haemodynamic stress in selected subtypes of patients with moyamoya disease: a positron emission tomography study. J Neurol Neurosurg Psychiatry 76:663-669, 2005

63. Nariai T, Senda M, Ishii K, Wakabayashi S, Yokota T, Toyama $\mathrm{H}$, et al: Posthyperventilatory steal response in chronic cerebral hemodynamic stress: a positron emission tomography study. Stroke 29:1281-1292, 1998

64. Nariai T, Suzuki R, Hirakawa K, Maehara T, Ishii K, Senda M: Vascular reserve in chronic cerebral ischemia measured by the acetazolamide challenge test: comparison with positron emission tomography. AJNR Am J Neuroradiol 16:563-570, 1995

65. Nariai T, Suzuki R, Matsushima Y, Ichimura K, Hirakawa K, Ishii K, et al: Surgically induced angiogenesis to compensate for hemodynamic cerebral ischemia. Stroke 25:1014-1021, 1994

66. Neff KW, Horn P, Schmiedek P, Düber C, Dinter DJ: 2D cine phase-contrast MRI for volume flow evaluation of the brain-supplying circulation in moyamoya disease. AJR Am J Roentgenol 187:W107-W115, 2006

67. Ogawa S, Clauss M, Kuwabara K, Shreeniwas R, Butura C, Koga S, et al: Hypoxia induces endothelial cell synthesis of membrane-associated proteins. Proc Natl Acad Sci U S A 88:9897-9901, 1991

68. Ohta T, Tanaka H, Kuroiwa T: Diffuse leptomeningeal enhancement, "ivy sign," in magnetic resonance images of moyamoya disease in childhood: case report. Neurosurgery 37:1009-1012, 1995

69. Oshima H, Katayama Y, Hirayama T: Intracerebral steal phenomenon associated with global hyperemia in moyamoya disease during revascularization surgery. J Neurosurg 92:949954,2000 


\section{S. Bacigaluppi et al.}

70. Perren F, Horn P, Vajkoczy P, Schmiedek P, Meairs S: Power Doppler imaging in detection of surgically induced indirect neoangiogenesis in adult moyamoya disease. J Neurosurg 103:869-872, 2005

71. Rostrup E, Larsson HB, Toft PB, Garde K, Thomsen C, Ring $\mathrm{P}$, et al: Functional MRI of $\mathrm{CO} 2$ induced increase in cerebral perfusion. NMR Biomed 7:29-34, 1994

72. Ruan LT, Duan YY, Cao TS, Zhuang L, Huang L: Color and power Doppler sonography of extracranial and intracranial arteries in Moyamoya disease. J Clin Ultrasound 34:60-69, 2006

73. Sakamoto S, Ohba S, Shibukawa M, Kiura Y, Arita K, Kurisu $\mathrm{K}$ : CT perfusion imaging for childhood moyamoya disease before and after surgical revascularization. Acta Neurochir (Wien) 148:77-81, 2006

74. Sato S, Shirane R, Maruoka S, Yoshimoto T: Evaluation of neuronal loss in adult moyamoya disease by 123I-iomazenil SPECT. Surg Neurol 51:158-163, 1999

75. Schreiber WG, Gückel F, Stritzke P, Schmiedek P, Schwartz A, Brix G: Cerebral blood flow and cerebrovascular reserve capacity: estimation by dynamic magnetic resonance imaging. J Cereb Blood Flow Metab 18:1143-1156, 1998

76. Shimizu H, Shirane R, Fujiwara S, Takahashi A, Yoshimoto T: Proton magnetic resonance spectroscopy in children with moyamoya disease. Clin Neurol Neurosurg 99 (2 Suppl): S64-S67, 1997

77. Suzuki J, Takaku A: Cerebrovascular "moyamoya" disease. Disease showing abnormal net-like vessels in base of brain. Arch Neurol 20:288-299, 1969

78. Takahashi M, Miyauchi T, Kowada M: Computed tomography of Moyamoya disease: demonstration of occluded arteries and collateral vessels as important diagnostic signs. Radiology 134:671-676, 1980

79. Takase K, Kashihara M, Hashimoto T: Transcranial Doppler ultrasonography in patients with moyamoya disease. Clin Neurol Neurosurg 99 (2 Suppl):S101-S105, 1997

80. Takeuchi S, Kobajashi K, Tuchida T, Imamura H, Tanaka R, Ito J: Computed tomography in Moyamoya disease. J Comput Assist Tomogr 6:24-32, 1982

81. Tosaka M, Sato N, Fujimaki H, Tanaka Y, Kagoshima K, Takahashi A, et al: Diffuse pachymeningeal hyperintensity and subdural effusion/hematoma detected by fluid-attenuated inversion recovery MR imaging in patients with spontaneous intracranial hypotension. AJNR Am J Neuroradiol 29:1164-1170, 2008

82. Touho H, Karasawa J, Ohnishi H: Preoperative and postoperative evaluation of cerebral perfusion and vasodilatory capacity with 99mTc-HMPAO SPECT and acetazolamide in childhood Moyamoya disease. Stroke 27:282-289, 1996
83. van Straten M, Venema HW, Majoie CB, Freling NJ, Grimbergen CA, den Heeten GJ: Image quality of multisection CT of the brain: thickly collimated sequential scanning versus thinly collimated spiral scanning with image combining. AJNR Am J Neuroradiol 28:421-427, 2007

84. Vesely A, Sasano H, Volgyesi G, Somogyi R, Tesler J, Fedorko L, et al: MRI mapping of cerebrovascular reactivity using square wave changes in end-tidal PCO2. Magn Reson Med 45:1011-1013, 2001

85. Vörös E, Kiss M, Hankó J, Nagy E: Moyamoya with arterial anomalies: relevance to pathogenesis. Neuroradiology 39:852-856, 1997

86. Warren DJ, Hoggard N, Walton L, Radatz MW, Kemeny AA, Forster DM, et al: Cerebral arteriovenous malformations: comparison of novel magnetic resonance angiographic techniques and conventional catheter angiography. Neurosurgery 61 (1 Suppl): 187-196, 2007

87. Wintermark M, Maeder P, Verdun FR, Thiran JP, Valley JF, Schnyder P, et al: Using $80 \mathrm{kVp}$ versus $120 \mathrm{kVp}$ in perfusion CT measurement of regional cerebral blood flow. AJNR Am J Neuroradiol 21:1881-1884, 2000

88. Wintermark M, Sesay M, Barbier E, Borbély K, Dillon WP, Eastwood JD, et al: Comparative overview of brain perfusion imaging techniques. Stroke 36:e83-e99, 2005

89. Wintermark M, Sincic R, Sridhar D, Chien JD: Cerebral perfusion CT: technique and clinical applications. J Neuroradiol 35:253-260, 2008

90. Yamada I, Nakagawa T, Matsushima Y, Shibuya H: High-resolution turbo magnetic resonance angiography for diagnosis of Moyamoya disease. Stroke 32:1825-1831, 2001

91. Yang CY, Chen YF, Lee CW, Huang A, Shen Y, Wei C, et al: Multiphase CT angiography versus single-phase CT angiography: comparison of image quality and radiation dose. AJNR Am J Neuroradiol 29:1288-1295, 2008

92. Yang SH, Li B, Wang CC, Zhao JZ: Angiographic study of moyamoya disease and histological study in the external carotid artery system. Clin Neurol Neurosurg 99 (2 Suppl): S61-S63, 1997

93. Yonas H, Smith HA, Durham SR, Pentheny SL, Johnson DW: Increased stroke risk predicted by compromised cerebral blood flow reactivity. J Neurosurg 79:483-489, 1993

Manuscript submitted December 14, 2008.

Accepted January 26, 2009.

Address correspondence to: Susanna Bacigaluppi, M.D., Division of Neurosurgery, Toronto Western Hospital, WW4-450, 399 Bathurst Street, Toronto, Ontario, Canada M5T 2S8. email: susannabacigaluppi@yahoo.it. 